\title{
Esperança de vida de pessoas com câncer acompanhadas pela Atenção Primária à
}

\section{Saúde}

\author{
Hope of life of people with cancer accompanied by Primary Health Care \\ Esperanza de vida de las personas com cáncer monitoreadas por la Atención Primaria de Salud
}

Recebido: 07/03/2021 | Revisado: 14/03/2021 | Aceito: 25/03/2021 | Publicado: 02/04/2021

Renata Ramos Menezes

ORCID: https://orcid.org/0000-0003-0122-2863 Universidade Federal de Sergipe, Brasil

E-mail: renata.ramos.menezes@hotmail.com

Simone Yuriko Kameo

ORCID: https://orcid.org/0000-0002-0035-2415 Universidade Federal de Sergipe, Brasil E-mail: simonekameo@hotmail.com

Dayane Ketlyn da Cunha Santos

ORCID: https://orcid.org/0000-0002-9312-4891 Universidade Federal de Sergipe, Brasil

E-mail: daayketlyn27@gmail.com

Karoline Alves de Almeida

ORCID: https://orcid.org/0000-0002-3078-5465 Universidade Federal de Sergipe, Brasil E-mail: karolinealvesalm@gmail.com

Lucas Pereira Santos

ORCID: https://orcid.org/0000-0002-2506-1761 Universidade Federal de Sergipe, Brasil

E-mail: lucasprs05@gmail.com

Laislaine de Jesus Santos

ORCID: https://orcid.org/0000-0002-1887-3964 Universidade Federal de Sergipe, Brasil E-mail: laislaine_santos_@outlook.com

Thiago dos Santos Valença

ORCID: https://orcid.org/0000-0003-4554-8494 Universidade Federal de Sergipe, Brasil

E-mail: thiagovalenca.valena77@gmail.com Gabriel Agra Alencar Mocó

ORCID: https://orcid.org/0000-0002-4469-3123 Universidade Federal de Sergipe, Brasil E-mail: gabrielagramoco@gmail.com

\section{Resumo}

A esperança tem grande impacto na forma como o paciente lida com os problemas físicos, emocionais e espirituais. Desse modo, percebe-se que a esperança concede forças para que o paciente continue com a luta contra a sua condição. Objetivou-se mensurar a esperança de pessoas em tratamento ou seguimento do câncer, acompanhadas pela Atenção Primária à Saúde de Lagarto, Sergipe. Trata-se de uma pesquisa transversal e exploratória, de natureza quantitativa, com abordagens descritiva e analítica. Para a coleta dos dados, foram realizadas entrevistas com pessoas com diagnóstico de câncer através do instrumento de avaliação de esperança, a Escala de Esperança de Herth (EEH). A amostra foi composta por 42 pessoas, sendo que 47,6\% estavam em tratamento oncológico e 52,4\% em acompanhamento. O escore médio na Escala de Esperança de Herth foi 40,59, o que representa um alto nível de esperança, principalmente quando a esperança é associada à espiritualidade/religiosidade. É fundamental conhecer o que impacta negativamente nas variáveis acerca da esperança para proporcionar uma melhor qualidade de vida, uma vez que a esperança auxilia durante todo o percurso de enfrentamento ao câncer.

Palavras-chave: Neoplasias; Esperança; Adaptação psicológica; Atenção primária à saúde.

\begin{abstract}
The hope has an amazing impact how the pacient deal his physical, emotions and spirituals problems. Thus, can to perceive that the hope gives forces to pacientes fighting against their condicion. The objective was to quantify the people's hope in treatement or pursuance of cancer followed for the primary health care system in the city of Lagarto, Sergipe. This was a crosssectional, exploratory, quantitative study involving descriptive and analytical approaches. Data were collected through interviews with individuals diagnosed with cancer through holp instrument of avaliation, Herth Hope Scale (HHS). The study sample comprised 42 cancer people, 47,6\% in oncological treatment and 52,4\%
\end{abstract}


in accompaniment. The middle score in Herth Hope Scale was 40,59, that mean an high level of hope, mainly when the hope is associated with spirituality/religiosity. It is important to know what impacts harmfully in variables about hope to improve the quality of life, since the hope helps for only process of coping with cancer.

Keywords: Neoplasms; Hope; Adaptation psychological; Primary health care.

\section{Resumen}

La esperanza tiene un gran impacto en la manera como el paciente trata los problemas físicos, emocionales y espirituales. Así, se percibe que la esperanza dar fuerza al paciente para seguir luchando contra su condición. El objetivo fue medir la esperanza de las personas en tratamiento o seguimiento del cáncer, monitoreadas por la Atención Primaria de Salud de Lagarto, Sergipe. Se trata de una investigación transversal y exploratoria, de naturaleza cuantitativa, con enfoques descriptivos y analíticos. Para recopilar los datos, se llevaron a cabo entrevistas con personas diagnosticadas con cáncer mediante el instrumento de evaluación de la esperanza, la Escala de Esperanza de Herth (EEH). La muestra se compuso de 42 personas, de las cuales el 47,6\% estaban en tratamiento oncológico y el $52,4 \%$ eran monitoreadas. El puntaje promedio en la Escala de Esperanza de Herth fue 40,59, lo que representa un alto nivel de esperanza, especialmente cuando la esperanza se asocia con la espiritualidad/religiosidad. Es fundamental conocer los factores que impactan negativamente las variables sobre la esperanza para ofrecer una mejor calidad de vida, ya que la esperanza ayuda a lo largo de la trayectoria de afrontamiento del cáncer.

Palabras clave: Neoplasias; Esperanza; Adaptación psicológica; Atención primaria de salud.

\section{Introdução}

A descoberta de uma doença oncológica desencadeia uma série de emoções que, na maioria das vezes, entram em conflito com tudo o que alguém pensou para sua vida. O futuro torna-se incerto e, assim, podem surgir ideias de sofrimento, tanto pessoal como familiar, dependência, incapacidade e até de uma possível morte prematura (Cardoso, Luengo, Trancas, Vieira \& Reis, 2009). A coexistência de outras doenças intensifica esse processo, como os distúrbios psiquiátricos, que podem reforçar a dor e a incapacidade do paciente. Além disso, o humor pode oscilar e afetar os relacionamentos entre o indivíduo com câncer e os seus cuidadores (Azevedo, Pessalacia, Mata, Zoboli \& Pereira, 2017).

Diante disso, no campo da saúde, a esperança é vista como um desejo de que um evento futuro se realize, tem influência positiva no bem-estar e reflete um estado de positividade. Diversos estudos mostram que a esperança tem grande impacto na forma como o paciente lida com os problemas físicos, emocionais e espirituais (Olver, 2012). Desse modo, percebe-se que a esperança concede forças para que o paciente continue com a luta contra a sua condição, o enfrentamento das rotinas de tratamento e a mudança de seu estilo de vida (Sartore \& Grossi, 2008).

Nesse contexto, o apoio emocional, baseado na comunicação, torna-se indispensável para um melhor cuidado e, consequentemente, melhor resposta terapêutica. No entanto, é importante destacar que, cada indivíduo enfrenta sua condição de forma individual (Cardoso et al., 2009). Além do apoio emocional, os suportes espiritual e familiar são meios de enfrentamento que favorecem o despertar e a permanência da esperança.

Esses meios de enfrentamento podem ser utilizados para que um paciente apresente a esperança em meio a sua doença, já que se trata de um sentimento que impulsiona o ser humano em seu existir e possibilita o sentimento de um futuro positivo (Sartore \& Grossi, 2008). Destaca-se o papel da religiosidade, da espiritualidade e da fé, uma vez que geram esperança, propósito e significado, além de possibilitarem a criação de mecanismos para lidar com a doença (Figueiredo, Souza, Coelho \& Souza, 2018).

Assim, o presente estudo objetivou mensurar a esperança de pessoas em tratamento ou seguimento do câncer, acompanhadas pela Atenção Primária à Saúde (APS) do município de Lagarto, localizado no estado de Sergipe.

\section{Metodologia}

Trata-se de uma pesquisa transversal e exploratória, de natureza quantitativa, com abordagens descritiva e analítica, cuja amostra foi não probabilística e por conveniência. Para um maior rigor metodológico, o estudo teve como referência a 
Iniciativa STROBE (Malta, Cardoso, Bastos, Magnanini \& Silva, 2010). Foi realizado o levantamento das pessoas com câncer nas Unidades Básicas de Saúde (UBS) de Lagarto/SE por meio de relatos das equipes e consulta dos prontuários, uma vez que não há um sistema de informação sobre os pacientes oncológicos na APS do município.

Os critérios de inclusão compreenderam: pessoas com diagnóstico de câncer vinculadas às áreas das UBS do município, maiores de 18 anos, com diagnóstico de câncer confirmado por histologia ou citologia, realizando e/ou que tenham realizado qualquer modalidade de tratamento oncológico regular (quimioterapia e radioterapia, a partir do primeiro ciclo, cirurgia e hormonioterapia), em acompanhamento clínico e com condições cognitivas satisfatórias para responder sozinho aos questionários selecionados.

A princípio, 71 pessoas foram selecionadas para a pesquisa. Destas, quatro recusaram-se a participar; duas mantinham em sigilo a doença; duas eram crianças; oito não foram encontradas pelos agentes comunitários em saúde (ACS) em suas casas; três não iniciaram o tratamento durante o período da coleta; cinco não foram entrevistados por dificuldade no contato com os ACS e cinco apresentaram dificuldades cognitivas durante as entrevistas. Ao final, a amostra foi composta por 42 pessoas com câncer.

A partir da análise do levantamento de dados, as pessoas foram identificadas e selecionadas, conforme os critérios de inclusão, as entrevistas nos domicílios foram agendadas, com a ajuda dos ACS, e realizadas de acordo com a disponibilidade dos envolvidos.

Para a coleta dos dados, foram realizadas entrevistas face a face, e utilizada a Escala de Esperança de Herth (EEH) adaptada e validada para a língua portuguesa (Sartore \& Grossi, 2008). Essa escala originou-se do instrumento específico norte-americano para mensuração da esperança, chamado Herth Hope Index (Sartore \& Grossi, 2008; Wakiuchi, Marchi, Norvila, Marcon \& Sales, 2015). Utilizada em vários contextos, como nos casos de recidiva de câncer, intervenção no serviço de enfermagem e até mesmo para avaliar idosos, a EEH possui a função de complementar o cuidado e melhorar a qualidade de vida do paciente, de maneira a entender suas perspectivas quanto ao futuro (Sartore \& Grossi, 2008).

A Escala de Esperança de Herth consiste em 12 itens originais escritos de forma afirmativa, na qual a graduação dos itens ocorre por escala tipo Likert de 4 pontos. As opções estão entre concordo completamente (4), concordo (3), discordo (2) e discordo completamente (1). As afirmações 3 e 6 apresentam escores invertidos, e o escore total varia de 12 a 48. Quanto maior o escore, mais alto o nível de esperança. Esses itens abordam questões como otimismo, fé, força, certeza e afeto, sendo pontos importantes para a manutenção da esperança (Sartore \& Grossi, 2008).

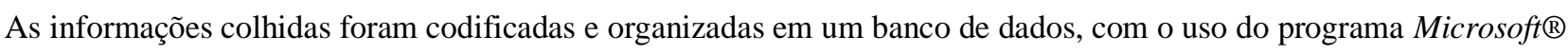
Office Excelß (2016), e também foi adotada a análise estatística através do programa SPSS (Statistical Package For The Social Science versão 22.0 para Windows). Dessa forma, realizou-se uma estatística descritiva em frequência absoluta, medidas de posição (média, mediana, mínima e máxima) e de dispersão (desvio padrão). Além disso, para testar a confiabilidade da Escala de Esperança de Herth foi executado o teste de consistência interna alfa de Cronbach.

O projeto de pesquisa foi aprovado pelo Comitê de Ética em Pesquisa (CEP) da Universidade Federal de Sergipe (UFS), com número do Parecer 1.917.297. Foram seguidas diretrizes e normas regulamentadoras preconizadas na resolução ${ }^{\circ}$ 466/2012 do Conselho Nacional de Saúde (CNS) sobre pesquisas que envolvem seres humanos.

\section{Resultados}

Dentre as 42 pessoas com câncer entrevistadas, 52,4\% eram do sexo feminino e 47,6\% do sexo masculino, e com a faixa etária entre 40 a 99 anos, sendo a maior prevalência entre 70 a 79 anos $(28,6 \%)$ e média de 65,45 anos. A maioria dos participantes eram pardos $(45,2 \%)$, casados $(50 \%)$, com ensino fundamental incompleto $(52,4 \%)$, aposentados $(59,5 \%)$ e que 
moravam na zona urbana (83,3\%). As religiões relatadas entre os participantes foram católica (78,6\%), evangélica (19\%) e adventista $(2,4 \%)$.

Em relação às características clínicas, 47,6\% estavam em tratamento oncológico (47,6\%) e 52,4\% em acompanhamento (52,4\%). Os tipos de câncer mais relatados abrangeram próstata $(23,8 \%)$, mama (19\%) e pele $(14,3 \%)$. A recidiva da doença esteve presente em $4,8 \%$ dos participantes. Os tratamentos mencionados pelos participantes foram cirurgia, quimioterapia, radioterapia e hormonioterapia, sendo tratamentos isolados ou combinados.

O tempo de diagnóstico estava entre menor que 1 ano (28,6\%), 1 a 2 anos (14,3\%), 2 a 3 anos (2,4\%), 3 a 4 anos $(21,4 \%)$ e maior que 4 anos $(33,3 \%)$. O tempo de tratamento (participantes em tratamento) estava entre menor que 1 mês (10\%), 1 mês a 1 ano (35\%) e maior que 1 ano (55\%).

O Gráfico 1 descreve a frequência das variáveis da Escala de Esperança de Herth em pacientes oncológicos acompanhados pela Atenção Primária à Saúde.

Gráfico 1 - Frequência das variáveis da Escala de Esperança de Herth em pacientes oncológicos acompanhados pela Atenção Primária à Saúde na cidade de Lagarto, Sergipe, Brasil.

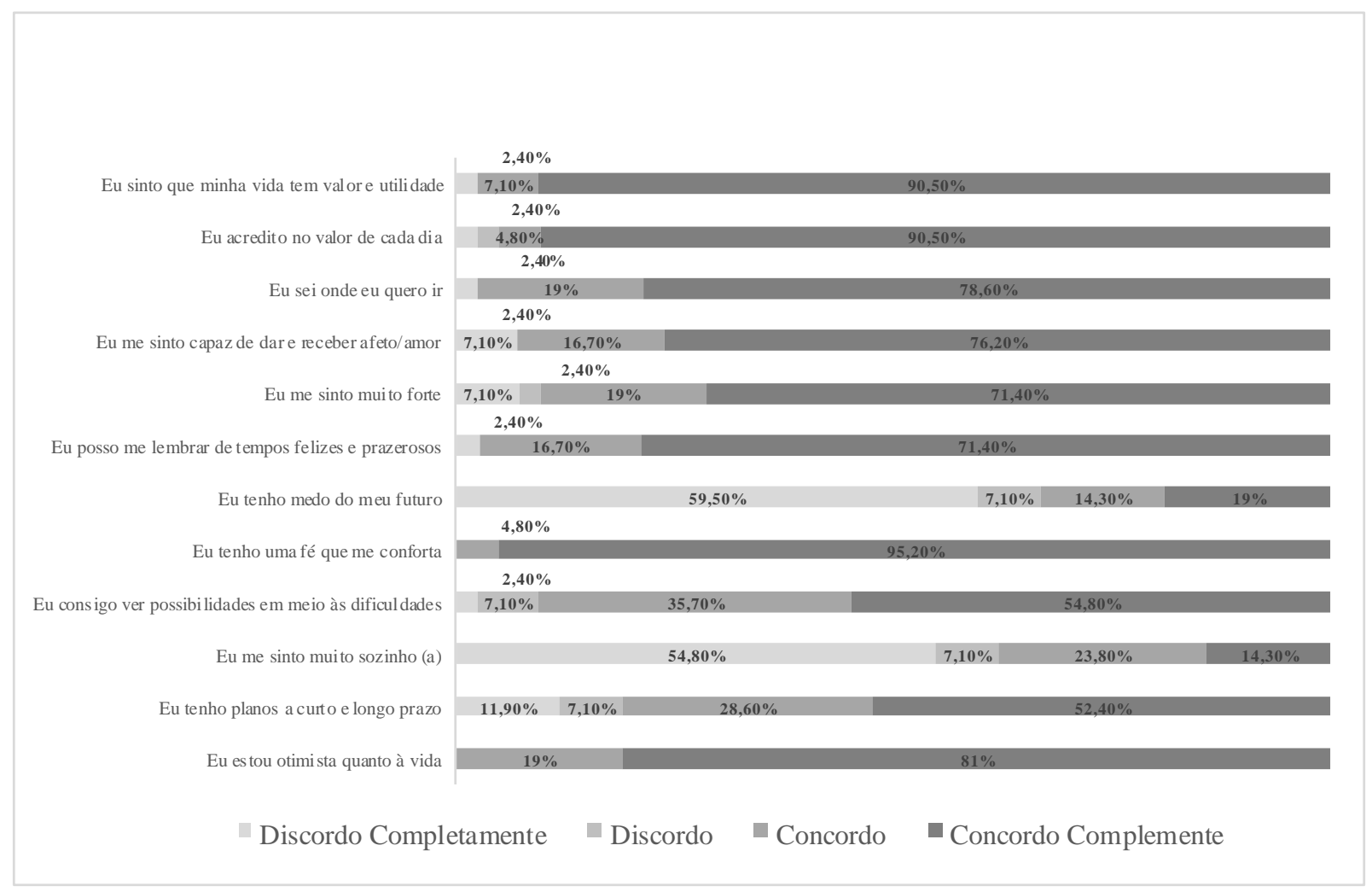

Fonte: Autores.

O gráfico acima mostra que, grande parte dos pacientes analisados apresentaram-se positivos e esperançosos, pois as variáveis relacionadas à valorização da vida, otimismo, força revelam níveis de concordância acima de 70\%. No entanto, os níveis de discordância da variável que se refere à solidão também são elevados. Ademais, destaca-se ainda que, 95,20\% dos pacientes possuíam uma "fé que conforta", o que demonstra a importância da espiritualidade na manutenção da esperança nos pacientes oncológicos.

A Tabela 1 descreve a análise descritiva das variáveis da Escala de Esperança de Herth em pacientes oncológicos em relação à média, desvio padrão, mediana, variação obtida e variação esperada. 
Tabela 1 - Análise descritiva das variáveis da Escala de Esperança de Herth em pacientes oncológicos acompanhados pela Atenção Primária à Saúde na cidade de Lagarto, Sergipe, Brasil.

\begin{tabular}{c|c|c|c|c|c}
\hline Variável & Média & $\begin{array}{c}\text { Desvio- } \\
\text { Padrão }\end{array}$ & Mediana & $\begin{array}{c}\text { Variação } \\
\text { obtida }\end{array}$ & $\begin{array}{c}\text { Variação } \\
\text { esperada }\end{array}$ \\
\hline Eu estou otimista quanto à vida & 3,80 & 0,39 & 4,0 & $3-4$ & $1-4$ \\
Eu tenho planos a curto e longo prazo & 3,21 & 1,02 & 4,0 & $1-4$ & $1-4$ \\
Eu me sinto muito sozinho (a) & 1,97 & 1,17 & 1,0 & $1-4$ & $1-4$ \\
Eu consigo ver possibilidades em meio às dificuldades & 3,42 & 0,73 & 4,0 & $1-4$ & $1-4$ \\
Eu tenho uma fé que me conforta & 3,95 & 0,21 & 4,0 & $3-4$ & $1-4$ \\
Eu tenho medo do meu futuro & 1,92 & 1,23 & 1,0 & $1-4$ & $1-4$ \\
Eu me sinto muito forte & 3,76 & 0,57 & 4,0 & $1-4$ & $1-4$ \\
Eu posso me lembrar de tempos felizes e prazerosos & 3,54 & 0,86 & 4,0 & $1-4$ & $1-4$ \\
Eu me sinto capaz de dar e receber afeto/amor & 3,61 & 0,82 & 4,0 & $1-4$ & $1-4$ \\
Eu sei onde eu quero ir & 1,00 & 0,58 & 4,0 & $1-4$ & $1-4$ \\
Eu acredito no valor de cada dia & 3,83 & 0,58 & 4,0 & $1-4$ & $1-4$ \\
Total & 3,85 & 0,52 & 4,0 & $1-4$ & $1-4$ \\
\hline
\end{tabular}

Fonte: Autores.

Na Tabela 1, observa-se que a variação obtida foi menor nas variáveis "Eu estou otimista quanto à vida" e "Eu tenho uma fé que me conforta", sendo a variação de 3-4, o que significa que a amostra comportou-se positivamente, de forma homogênea, com prevalência de, respectivamente, $81 \%$ e 95,2\% da resposta "concordo completamente". As demais perguntas, por sua vez, apresentaram variação obtida similar à esperada (1-4). Ao analisar o desvio-padrão, foi possível perceber que as variáveis "Eu tenho medo do meu futuro", "Eu me sinto muito sozinho (a)" e "Eu tenho planos a curto e longo prazo" apresentaram maiores valores, o que indica maior discordância entre as respostas dos participantes.

Diante das maiores e menores médias das afirmações, boa parte dos entrevistados pontuaram positivamente na Escala de Esperança de Herth, principalmente quando a esperança foi associada à espiritualidade/religiosidade.

O alfa de Cronbach estima a consistência interna e, consequentemente, a confiabilidade do questionário aplicado em uma pesquisa. Assim, no presente estudo, o valor foi de 0,583.

\section{Discussão}

O escore médio na Escala de Esperança de Herth dos pacientes estudados foi 40,59, o que representa um alto nível de esperança. Esse resultado assemelha-se a resultados de dois estudos, o de Sartore e Grossi (2008) e o de Balsanelli, Grossi e Herth (2011), com média de 41,57 e 43 em pacientes oncológicos, respectivamente.

Os dados sociodemográficos mostram que, dentro da população estudada, a maioria correspondeu ao sexo feminino $(52,4 \%)$. Esse dado encontra-se em consonância o estudo de Grandizoli, Ibiapina, Júnior e Garcia (2017) que também verificou uma superioridade feminina (64\%) em relação ao público masculino (36\%). A pesquisa de Viana, Querido, Dixe e Barbosa (2010) também demonstrou 56,4\% de mulheres no total dos entrevistados. Os estudos de Balsanelli et al. (2011) e Macêdo, Gomes e Bezerra (2019) tiveram como público-alvo apenas mulheres por tratarem da avaliação da esperança em pacientes com câncer de mama. 
Em relação à faixa etária, a média foi de 65,45 anos, sendo um resultado semelhante à pesquisa de Viana et al. (2010), cuja média de idade foi de 67,3 anos.

A esperança, quando em níveis elevados, é considerada como ferramenta que proporciona maior capacidade de enfrentamento às dificuldades e melhor resolutividade frente aos problemas. Diante disso, a esperança está relacionada a perspectivas positivas quanto ao futuro e impacta diretamente na qualidade de vida (Sartore \& Grossi, 2008). É possível estabelecer essa relação, tendo em vista os dados que revelam as respostas dos pacientes com altas pontuações em afirmações associadas ao otimismo, planos a curto e longo prazo, possibilidades diante das dificuldades, lembranças de tempos felizes e prazerosos, sobre sentir-se forte e sentir que a própria vida é valiosa. Assim, esses resultados corroboram com o relato de um paciente do estudo de Sales, Cassarotti, Piolli, Matsuda e Wakiuchi (2015), o qual afirmou sobre a esperança ser fundamental em todas as situações da vida.

Destaca-se que, uma das variáveis de menor média deste estudo corresponde à afirmação "Eu me sinto muito sozinho" (média de 1,49), em que apresenta escore invertido. Essa variável foi a de menor média encontrada na pesquisa de Wakiuchi et al. (2015) com pacientes oncológicos, cuja média foi de $2,67( \pm 1,05)$ e 2,57 pontos $( \pm 1,01)$ durante e depois do fim do tratamento quimioterápico, respectivamente.

O tempo de diagnóstico e tratamento em que a EEH foi aplicada é de suma importância e deve ser analisado. No presente estudo, o tempo desde o diagnóstico de boa parte dos pacientes $(33,3 \%)$ foi de mais de 4 anos, e o tempo de tratamento (participantes em tratamento), maior que 1 ano (55\%). Nesse sentido, o estudo de Wakiuchi et al. (2015) revelou que o nível de esperança aumentou no final do tratamento quimioterápico, mas não significativamente, devido à maior escolaridade, ausência de metástases, tratamento curativo ou adjuvante e ausência de dor moderada a forte. Outro estudo, por sua vez, demonstrou que os pacientes que mais demoraram a iniciar o tratamento quimioterápico apresentaram diminuição significativa nos escores de esperança ao longo do mesmo (Balsanelli et al., 2011), o que mostra o impacto do tempo de início do tratamento.

Uma ferramenta de apoio essencial é a família, em que é afetada pela doença e precisa desenvolver estratégias de suporte para readquirir equilíbrio durante o enfrentamento (Marcon et al., 2010). São notáveis as mudanças na dinâmica familiar quando há o surgimento do câncer, pois a família tenta se adaptar frente às novas condições para vivenciar um bemestar (Wernet \& Ângelo, 2003). Diante disso, há resultados positivos quanto às questões relacionadas à interação emocional, ao não se sentir solitário e a perspectivas de futuro, além da capacidade de dar e receber amor e afeto.

Mesmo a amostra da presente pesquisa ter revelado bons escores quando questionados sobre "sentir-se sozinho", é importante observar que, os pacientes podem mascarar a sua experiência de sofrimento, devido à preocupação com o bem-estar de outras pessoas. Os pacientes podem internalizar sentimentos bons e otimistas para que os que estão ao seu redor possam também apresentar os mesmos sentimentos (Sales et al., 2014). Desse modo, é preciso que os profissionais de saúde que acompanham o paciente se atentem para esse possível comportamento, uma vez que o sentimento de estigma pela doença e o temor de provocar sofrimentos às pessoas próximas podem prejudicar durante a adaptação psicológica à doença (Cardoso et al., 2009).

Além disso, fatores podem interferir na esperança da pessoa com câncer, como comentários em relação à aparência do paciente ou às vivências referentes a outras pessoas que tiveram a mesma doença, assim como mortes ou sequelas (Sales et al., 2014). Além disso, os próprios momentos de intensificação dos sintomas e a baixa autoestima podem causar angústia e deixar o paciente novamente desacreditado (Balsanelli et al., 2011). Sendo assim, ressalta-se que, quanto maior o escore de esperança, menor o escore de depressão, e, que a correlação entre esperança e autoestima é positiva, já que quanto maior a esperança, maior a autoestima (Balsanelli \& Grossi, 2016). Desse modo, é importante a esperança para o bem-estar emocional e mental do indivíduo. 
Deve-se dar ênfase também ao impacto da espiritualidade nos níveis de esperança, já que a média deste estudo na variável "Eu tenho uma fé que me conforta" foi de 3,95\%. Essa relevância foi também observada em uma pesquisa (Sales et al., 2014) que, diante das circunstâncias do câncer, o paciente procura a esperança em uma força maior, e essa força, muitas vezes, é percebida por auxílio da apreciação de sua fé ou crença em Deus. Assim, a espiritualidade apresenta-se como suporte estratégico para o paciente oncológico durante o enfrentamento, principalmente, em momentos difíceis, ao permitir o alívio da dor e do sofrimento, bem como por proporcionar esperança para vencer a doença, como demonstrou o estudo qualitativo de Rocha, Lima, Dias, Paiva e Rocha. (2016).

\section{Conclusão}

Esse estudo e os dados presentes na literatura demonstram que a esperança e a sua avaliação através da EEH contribuem efetivamente para a redução do impacto da doença no cotidiano do paciente. Sendo assim, é importante que essa escala continue a ser testada em diferentes contextos sociais e culturais brasileiros, pois conhecer o que impacta negativamente nas variáveis acerca da esperança é fundamental para proporcionar uma melhor qualidade de vida. Desse modo, os profissionais da saúde devem valorizar o sentimento de esperança, diante da avaliação e condutas necessárias no cuidado à pessoa com câncer.

Outrossim, foi possível perceber que os pacientes oncológicos da presente pesquisa apresentaram bons índices de esperança, com otimismo em relação à vida e ao valor de cada dia, bem como na utilidade de estar vivo, com capacidade de recordação de tempos felizes e prazerosos, e apresentar a fé como conforto e auxílio nos momentos difíceis.

A relevância do presente estudo configura-se no impacto da esperança para o paciente oncológico, uma vez que auxilia durante todo o percurso de enfrentamento ao câncer. É importante pontuar que, esse estudo apresentou limitações como o pequeno número de participantes e a seleção da amostra por conveniência, o que interfere em sua utilização em relação à população geral.

Diante disso, demonstra-se a necessidade de novos estudos que visem conhecer a esperança de pessoas com câncer em outras realidades, como durante a internação hospitalar ou cuidados paliativos, a fim de buscar mais alternativas para melhorar a qualidade de vida dos pacientes oncológicos.

\section{Referências}

Azevedo, C., Pessalacia, J. D. R., Mata, L. R. F., Zoboli, E. L.C. P. \& Pereira, M. G. (2017). Interface between social support, quality of life and depression in users eligible for palliative care. Revista da Escola de Enfermagem da USP, 51, e03245, 1-8. https://doi.org/10.1590/s1980-220x2016038003245.

Balsanelli, A. C. S., Grossi, S. A. A. \& Herth, K. (2011). Avaliação da esperança em pacientes com doença crônica e em familiares ou cuidadores. Acta Paulista de Enfermagem, 24(3), 354-358. https://dx.doi.org/10.1590/S0103-21002011000300008.

Balsanelli, A. C. S. \& Grossi, S. A. A. (2016). Preditores de esperança entre mulheres com câncer de mama durante quimioterapia. Revista da Escola de Enfermagem da USP, 50(6), 898-904. http://doi.org/10.1590/S0080-623420160000700004.

Cardoso, G., Luengo, A., Trancas, B., Vieira, C. \& Reis, D. (2009). Aspectos Psicológicos do Doente Oncológico. Revista do Serviço de Psiquiatria do Hospital Prof. Doutor Fernando Fonseca - EPE, 7, 8-18. https://doi.org/10.25752/psi.4007.

Figueiredo, J. F., Souza, V. M., Coelho, H. V. \& Souza, R. S. (2018). Qualidade de vida de pacientes oncológicos em cuidados paliativos. Revista de Enfermagem do Centro-Oeste Mineiro, 8. https://doi.org/10.19175/recom.v8i0.2638.

Grandizoli, M. V., Ibiapina, I. S. M., Júnior, R. S. \& Garcia, V. C. B. (2017). Indicadores de esperança, ansiedade e depressão de pacientes em tratamento oncológico. Arquivos de Ciências da Saúde, 24(3), 65-70. https://doi.org/10.17696/2318-3691.24.3.2017.718.

Macêdo, E. L., Gomes, E. T. \& Bezerra, S. M. M. S. (2019). Esperança de Mulheres em tratamento quimioterápico para o câncer de mama. Cogitare Enfermagem, 24. http://dx.doi.org/10.5380/ce.v24i0.65400.

Malta, M., Cardoso, L. O., Bastos, F. I., Magnanini, M. M. F. \& Silva, C. M. F. P. (2010). Iniciativa STROBE: subsídios para a comunicação de estudos observacionais. Revista de Saúde Pública, 44(3), 559-565. https://doi.org/10.1590/S0034-89102010000300021. 
Research, Society and Development, v. 10, n. 4, e12510413644, 2021

(CC BY 4.0) | ISSN 2525-3409 | DOI: http://dx.doi.org/10.33448/rsd-v10i4.13644

Marcon, S. S., Radovanovic, C. A. T., Salci, M. A., Carreira, L., Haddad, M. L. \& Faquinello, P. (2010). Estratégias de cuida do a famílias que convivem com a doença crônica em um de seus membros. Cuidado E Saúde, 8, 70-78. http://doi.org/10.4025/cienccuidsaude.v8i0.9720.

Olver, I. N. (2012). Evolving definitions of hope in oncology. Current Opinion in Supportive and Palliative Care, 6(2), 236-241. https://doi.org/10.1097/SPC.0b013e3283528d0c.

Rocha, P. T., Lima, C. A., Dias, O. V., Paiva, P. A. \& Rocha, J. F. D. (2016). A Influência da Espiritualidade e da Religiosidade no Tratamento Oncológico: percepção da pessoa com câncer. Revista de Tendências da Enfermagem Profissional, 8(4), 2031-2036. <http://www.coren-ce.org.br/wpcontent/uploads/2019/03/A-INFLU\%C3\%8ANCIA-DA-ESPIRITUALIDADE-E-DA-RELIGIOSIDADE-NO-TRATAMENTO-ONCOL\%C3\%93GICO.pdf>.

Sales, C. A., Cassarotti, M. S., Piolli, K. C., Matsuda, L. M. \& Wakiuchi, J. (2014). O sentimento de esperança em pacientes com câncer: uma análise existencial. Revista da Rede de Enfermagem do Nordeste, 15(4), 659-667. http://doi.org/10.15253/2175-6783.2014000400013.

Sartore, A. C. \& Grossi, S. A. A. (2008). Escala de Esperança de Herth: instrumento adaptado e validado para a língua portuguesa. Revista da Escola de Enfermagem da USP, 42(2), 227-232. http://doi.org/10.1590/S0080-62342008000200003.

Viana, A., Querido, A., Dixe, M. A. \& Barbosa, A. (2010). Avaliação da esperança em cuidados Paliativos. Revista INFAD de Psicologia, 2, 607-616. http://infad.eu/RevistaINFAD/index.php/publicaciones/revista-infad-2010/no1-volumen-2-indice/.

Wakiuchi, J., Marchi, J. A., Norvila, L. S., Marcon, S. S. \& Sales, C. A. (2015). Esperança de vida de pacientes com câncer submetidos à quimioterapia. Acta Paulista de Enfermagem, 28(3), 202-208. https://doi.org/http://dx.doi.org/10.1590/1982- 0194201500035.

Wernet, M. \& Ângelo, M. (2003). Mobilizando-se para a família: dando um novo sentido à família e ao cuidar. Revista da Escola de Enfermagem da USP, 37, 19-25. http://doi.org/10.1590/S0080-62342003000100003. 